\title{
Review
}

Fetal Diagnosis

and Therapy

Fetal Diagn Ther 2017;42:1-8

DOI: $10.1159 / 000455947$
Received: September 5, 2016

Accepted after revision: January 3, 2017

Published online: May 9, 2017

\section{Fetal Tricuspid Regurgitation in the First Trimester as a Screening Marker for Congenital Heart Defects: Systematic Review and Meta-Analysis}

\author{
Carolina Scala ${ }^{a-c}$ Maddalena Morlando ${ }^{c, d} \quad$ Alessandra Familiari $^{c}$ \\ Umberto Leone Roberti Maggiore $^{a, b}$ Simone Ferrero ${ }^{a, b} \quad$ Francesco D'Antonio $^{c}$ \\ Asma Khalilc \\ ${ }^{a}$ Academic Unit of Obstetrics and Gynaecology, IRCCS AOU San Martino - IST, and b Department of Neurosciences, \\ Rehabilitation, Ophthalmology, Genetics, Maternal and Child Health (DiNOGMI), University of Genoa, \\ Genoa, Italy; 'Fetal Medicine Unit, St George's Hospital, St George's University of London, London, UK; \\ ${ }^{\mathrm{d} D e p a r t m e n t}$ of Neuroscience, Reproductive Science and Dentistry, University of Naples Federico II, Naples, Italy
}

\section{Keywords}

Tricuspid regurgitation · First trimester · Congenital heart defect

\begin{abstract}
Background: Assessment of tricuspid flow has been reported to improve the performance of screening for aneuploidies and congenital heart defects (CHD). However, the performance of tricuspid regurgitation (TR) as a screening marker for CHD in euploid fetuses is yet to be established. The main aim of this meta-analysis was to establish the predictive accuracy of TR for CHD. Methods: MEDLINE, Embase, and the Cochrane Library were searched electronically utilizing combinations of the relevant medical subject heading for "fetus," "tricuspid regurgitation," and "first trimester." The outcomes explored were prevalence of TR in an euploid population, strength of association between TR and CHD, and predictive accuracy of TR for CHD in euploid fetuses. Summary estimates of sensitivity, specificity, positive and nega-
\end{abstract}

\section{KARGER}

(C) 2017 S. Karger AG, Basel

E-Mail karger@karger.com

www.karger.com/fdt tive likelihood ratios, and diagnostic odds ratio for the overall predictive accuracy of TR for the detection of CHD were computed using the hierarchical summary receiver-operating characteristics model. Results: A total of 452 articles were identified; 60 were assessed with respect to their eligibility for inclusion and a total of 4 studies were included in the study. TR was associated with an increased risk of CHD (RR: 9.6, 95\% Cl 2.8-33.5; $1^{2}$ : 92.7\%). The strength of association between TR and CHD persisted when considering fetuses at risk for CHD, such as those with increased nuchal translucency (RR: 7.2, 95\% CI 5.2-9.8; $1^{2}: 0 \%$ ), while TR did not show any association with CHD when detected in a population at low risk for cardiac defects (RR: 9.3, 95\% Cl 0.8-111.8; $\left.I^{2}: 93 \%\right)$. The overall diagnostic performance of TR in detecting CHD was poor in detecting CHD (sROC: 0.684, SE: 0.61) with a sensitivity of $35.2 \%(95 \% \mathrm{Cl} 26.9-44.1)$ and a specificity of $98.6 \%$ ( $95 \% \mathrm{Cl} 98.5-98.7)$. Detection of TR at the 11-14 weeks' scan showed a positive likelihood ratio of $7.2(95 \% \mathrm{Cl}$ 5.3-9.8) in detecting CHD when applied to a population at risk for CHD such as fetuses with an increased nuchal trans-

Dr. Carolina Scala, MD

Fetal Medicine Unit

St George's University of London

London SW17 0RE (UK)

E-Mail carolinascala@icloud.com 
lucency. Conclusion: The detection of TR in the first trimester increases the risk of CHD. However, isolated TR in the first trimester does not seem to be a strong predictor for CHD.

(C) 2017 S. Karger AG, Basel

\section{Introduction}

Congenital heart defects (CHD) account for one-third of all congenital anomalies and are the leading cause of infant mortality due to birth defects [1]. CHD are the most common congenital abnormality diagnosed in fetal life [2] and are complicated by stillbirth and neonatal death in approximately 5 and $20 \%$ of the affected cases, respectively $[3,4]$.

Fetal cardiac anatomical evaluation is usually performed during the second trimester of pregnancy, at the time of the anomaly scan. Antenatal ultrasound screening detects up to $60 \%$ of fetuses with CHD [5]. This means that a large proportion of $\mathrm{CHD}$ are not detected during the pregnancy [5]. Moreover, the detection of fetal CHD in the first trimester is even lower. A review of the published series with more than 1,000 cases from 1993 to 2008 , which included data from 36,237 pregnancies generated by eight centers, suggests that the overall detection rate of major congenital anomalies at 11-13 weeks is $29 \%$ [6]. The detection rate of CHD in the first trimester has been estimated at around 30\% [7].

It has been reported that the assessment of tricuspid flow can improve the performance of first-trimester screening for aneuploidies and CHD [8-10]. However, the performance of tricuspid regurgitation (TR) as a screening marker for CHD in euploid fetuses is yet to be established.

The primary aim of this systematic review was to ascertain the diagnostic performance of TR, as detected at the first-trimester scan, in detecting CHD prenatally; the secondary aim was to explore the strength of association between TR and CHD in euploid fetuses.

\section{Methods}

\section{Search Strategy}

A protocol was designed a priori according to recommendations for systematic review and meta-analysis [11-13]. MEDLINE (since inception), Embase (since inception), and the Cochrane Library (since inception), were searched electronically on December 8 , 2015. Combinations of the following relevant medical subject heading $(\mathrm{MeSH})$ terms, keywords, and word variants were used: "fetus," "tricuspid regurgitation," "first trimester" (online suppl. material 1; see www.karger.com/doi/10.1159/000455947 for all online suppl. material). Reference lists of relevant articles and reviews were hand-searched for additional reports. The search was limited to English language. The study was registered with the PROSPERO database (registration number: CRD42016036390, http://www.crd.york.ac.uk/PROSPERO).

\section{Study Selection and Outcomes Analyzed}

Studies were selected in a two-stage process. The first step was to assess eligibility based on the titles and abstracts. In the second step, the decision for final inclusion was made after interrogating the full papers. The studies were included according to the following criteria: prenatal ultrasound evaluation of TR, description of its prevalence, and correlation with the outcome (CHD). The following potential biases in the methodology of the included studies were evaluated: characteristics of the population, prevalence of $\mathrm{CHD}$ in the study population, ultrasound methodology, and gestational age at ultrasound.

The primary aim of this systematic review was to ascertain the diagnostic performance of TR, as detected at the first-trimester scan, in detecting CHD prenatally; the secondary aim was to explore the strength of association between TR and CHD.

These outcomes were ascertained both in the overall population of fetuses undergoing prenatal ultrasound assessment and in those considered to be at increased risk of CHD, based on either maternal or fetal characteristics, such as those with high nuchal translucency (NT).

The ultrasound methodology was evaluated, and only studies with a clear description of adequate technique were included. In order for the diagnosis of TR to be made, the magnification of the image should be such that the fetal thorax occupies most of the image, an apical four-chamber view of the fetal heart obtained, and a pulsed-wave Doppler sample volume of $2.0-3.0 \mathrm{~mm}$ positioned across the tricuspid valve, the angle to the direction of flow being less than $30^{\circ}$ from the direction of the interventricular septum. In the first trimester, TR is diagnosed if it is found during at least half of the systole and with a velocity of over $60 \mathrm{~cm} / \mathrm{s}$ [12]. The sweep speed should be high $(2-3 \mathrm{~cm} / \mathrm{s})$, so that the waveforms are widely spread for better assessment. The sample volume should be placed across the valve at least three times in an attempt to interrogate the complete valve, as the tricuspid valve could be insufficient in one or more of its three cusps [14].

\section{Data Extraction and Quality Assessment}

Two authors (C.S. and M.M.) reviewed all abstracts independently, and agreement concerning potential relevance was reached by consensus. The same two authors independently reviewed fulltext copies of relevant papers and extracted relevant data regarding the number of pregnancies, gestational age at ultrasound, and prevalence of TR in euploid fetuses with and without CHD. Only papers investigating an association between TR detected in the first trimester of pregnancy and $\mathrm{CHD}$ were included. If more than one study was published for the same cohort with identical endpoints, the report containing the most comprehensive information on the population was included to avoid overlapping populations. For those articles in which the relevant information was not reported but the methodology suggested that this information would have been recorded initially, the authors were contacted. Only full-text articles were considered eligible for inclusion. Case reports, case series with fewer than three cases, and conference abstracts were excluded.
2

Fetal Diagn Ther 2017;42:1-8 DOI: $10.1159 / 000455947$
Scala et al. 
The PRISMA statement was followed while reporting the Methods, Results, and Discussion sections of the current review. The Quality in Prognosis Studies (QUIPS) tool was used to assess the methodological quality of the included studies.

\section{Statistical Analysis}

Summary estimates of sensitivity, specificity, positive and negative likelihood ratios (LR+ and LR-), and diagnostic odds ratio (DOR) for the overall predictive accuracy of the first-trimester ultrasound diagnosis of TR for the detection of CHD were computed using the hierarchical summary receiver-operating characteristics (HSROC) model [15]. Rutter and Gatsonis HSROC parameterization was used because it models functions of sensitivity and specificity to define a summary ROC curve, and its hierarchical modelling strategy can be used for comparisons of test accuracy when there is variability in threshold between studies [16]. The DOR is defined as the ratio of the odds of the test being positive if the subject has a disease, relative to the odds of the test being positive if the subject does not have the disease, i.e., LR+/LR- [17].

However, when the number of studies is small, the uncertainty associated with the estimation of the shape parameter could be very high, and models may fail to converge. Thus, for all metaanalyses in which less than four study estimates could be pooled, the DerSimonian-Laird random-effect model was used.

Between-study heterogeneity was explored using the $\mathrm{I}^{2}$ statistic, which represents the percentage of between-study variation due to heterogeneity rather than chance. A value of $0 \%$ indicates no observed heterogeneity, whereas $\mathrm{I}^{2}$ values $\geq 50 \%$ indicate a substantial level of heterogeneity. Random-effects models were used to test the robustness of results [18].

Meta-analysis of proportions was used to quantify the prevalence of TR in the first trimester. The units of the meta-analysis were single comparisons of subjects with abnormal versus normal ultrasound signs (TR) in predicting CHD in euploid fetuses. Only studies reporting a direct assessment of the risk were meta-analyzed. The results were reported as relative risks (RR) for the outcome observed.

Potential publication bias was formally assessed using Egger's regression asymmetry test. Following specific indications for metaanalyses of diagnostic accuracy, we correlated individual study sample sizes with both sensitivity and specificity as measures of test accuracy [19]. Tests for funnel plot asymmetry were not used when the total number of publications included for each outcome was below 10. In this case, the power of the test is too low to distinguish chance from real asymmetry [17]. Statistical analysis was performed using StatsDirect (StatsDirect LtD, Altrincham, UK), Stata command metandi (Stata Corp., College Station, TX, USA; 2013), and Meta-Disc 1.4 1.4 (http://www.hrc.es/investigacion/metadisc_ en.htm). A $p$ value $<0.05$ was considered statistically significant.

\section{Results}

A total of 452 articles were identified; 60 were assessed with respect to their eligibility for inclusion and a total of 4 studies were included in the systematic review (Fig. 1). A list of the 56 studies which were excluded, together with the reason for their exclusion, is shown in online supple-

Fetal TR in the First Trimester as a

Screening Marker for CHD
Potentially relevant citations identified by searching MEDLINE Embase, Scopus, and the Cochrane Library including the Cochrane Database of Systematic Reviews (CDSR), Database of Abstracts of Reviews of Effects (DARE), and the Cochrane Central Register of Controlled Trials (CENTRAL) and by hand searching $(n=452)$

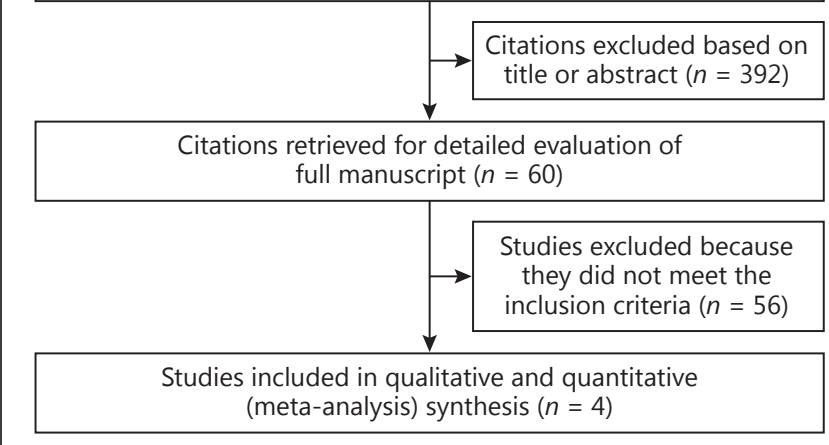

Fig. 1. Flowchart of the included studies.

mentary material 2. The general characteristics of the included studies are listed in Table 1. The quality assessment of the included studies using the QUIPS tool is shown in Table 2 . The risk of bias in the included studies was low in the different categories, including study participation, attrition, prognostic factor measurement, study confounding, and statistical analysis/reporting.

Of the four included studies, two were carried out in low-risk and two in high-risk populations; however, the study by Persico et al. [20] was performed in the same time interval of that of Faiola et al. [21]; thus it was not considered in the overall pooled analysis but only in the subanalysis including cases at risk for CHD such as those showing increased NT at the first-trimester scan.

Definition of high- and low-risk populations was different among the included studies. Wiechec et al. [22] analyzed an unselected population composed by all singleton gestations undergoing first-trimester screening for fetal aneuploidies. Pereira et al. [23] included cases with either normal or increased NT, although they excluded fetuses with noncardiac structural or chromosomal anomalies. In the study by Persico et al. [20], only women undergoing chorionic villus sampling were considered eligible for inclusion, while the study by Faiola et al. [21] considered only pregnancies referred to fetal echocardiography in view of the increased risk for aneuploidy based on the first-trimester screening results.

Euploid fetuses showing TR at the 11-14 weeks' scan had a significantly higher risk of having CHD compared to those without TR (RR: 9.6, 95\% CI 2.8-33.5; I²: 92.7\%).

Fetal Diagn Ther 2017;42:1-8 DOI: $10.1159 / 000455947$ 
Table 1. General characteristics of the studies included in the systematic review

\begin{tabular}{|c|c|c|c|c|}
\hline & Wiechec et al. [22] & Pereira et al. [23] & Persico et al. [20] & Faiola et al. [21] \\
\hline Year & 2015 & 2011 & 2011 & 2005 \\
\hline Country & Poland & UK & UK & UK \\
\hline Study design & retrospective & retrospective & prospective & retrospective \\
\hline Period considered & $2009-2012$ & $2006-2009$ & $2008-2009$ & $2001-2004$ \\
\hline Population analyzed & unselected & unselected & high risk & high risk \\
\hline FMF accreditation & yes & yes & yes & yes \\
\hline GA at scan & $11^{+0}-13^{+6}$ & $11^{+0}-13^{+6}$ & $11^{+0}-13^{+5}$ & $11^{+0}-13^{+6}$ \\
\hline Type of scan & TA and TV & TA & TA & TA \\
\hline Inclusion criteria & all CHD & $\begin{array}{l}\text { CHD requiring surgery or interventional } \\
\text { cardiac catheterization within the first } \\
\text { year of life }\end{array}$ & all CHD & $\begin{array}{l}\text { women referred for } \\
\text { fetal echocardiography }\end{array}$ \\
\hline Exclusion criteria & - & $\begin{array}{l}\text { aneuploidies and noncardiac defects } \\
\text { diagnosed prenatally or in the neonatal } \\
\text { period; pregnancies with no abnormal } \\
\text { fetal findings at the } 11-13 \text { weeks' scan, } \\
\text { the } 20-23 \text { weeks' scan, or both, which } \\
\text { resulted in termination, miscarriage, or } \\
\text { stillbirth }\end{array}$ & - & - \\
\hline CHD not considered & - & VSD, RAA, LSVC, ARSA, cardiac tumors & - & - \\
\hline Reference standard & $\begin{array}{l}\text { postnatal imaging, } \\
\text { autopsy findings }\end{array}$ & postnatal imaging, autopsy findings & $\begin{array}{l}\text { postnatal imaging, } \\
\text { autopsy findings }\end{array}$ & $\begin{array}{l}\text { postnatal imaging, } \\
\text { autopsy findings }\end{array}$ \\
\hline Fetuses, $n$ & 1,075 & 40,990 & 867 & 718 \\
\hline $\mathrm{CHD}, n$ & 11 & 85 & 100 & 149 \\
\hline
\end{tabular}

FMF, Fetal Medicine Foundation; GA, gestational age; CHD, congenital heart defect; TA, transabdominal; TV, transvaginal; VSD, ventricular septal defect; RAA, right aortic arch; LSVC, left superior vena cava; ARSA, aberrant right subclavian artery.

Table 2. Risk of bias in the included studies as assessed by the Quality in Prognostic Studies (QUIPS) tool

\begin{tabular}{|c|c|c|c|c|c|c|}
\hline \multirow[t]{2}{*}{ Study } & \multicolumn{6}{|c|}{ Level of risk of bias due to } \\
\hline & $\begin{array}{l}\text { study } \\
\text { participation }\end{array}$ & $\begin{array}{l}\text { study } \\
\text { attrition }\end{array}$ & $\begin{array}{l}\text { prognostic factor } \\
\text { measurement }\end{array}$ & $\begin{array}{l}\text { outcome } \\
\text { measurement }\end{array}$ & $\begin{array}{l}\text { study } \\
\text { confounding }\end{array}$ & $\begin{array}{l}\text { statistical analysis } \\
\text { and reporting }\end{array}$ \\
\hline Wiechec et al. [22] & low & low & low & low & low & low \\
\hline Pereira et al. [23] & low & low & low & low & low & low \\
\hline Persico et al. [20] & low & low & low & moderate & low & low \\
\hline Faiola et al. [21] & low & low & low & low & low & low \\
\hline
\end{tabular}

The strength of association between TR and CHD persisted when considering fetuses at risk for $\mathrm{CHD}$, such as those with increased NT (RR: 7.2, 95\% CI 5.2-9.8; $\mathrm{I}^{2}: 0 \%$ ), while TR did not show any association with CHD when detected in a population at low risk for cardiac defects (RR: 9.3, 95\% CI 0.8-111.8; I²: 93\%) (Fig. 2).

The overall diagnostic performance of TR in detecting CHD during the first trimester of pregnancies was assessed in three studies [21-23]; TR has an overall poor diagnostic accuracy in detecting $\mathrm{CHD}$ with an sROC of
0.684 (SE: 0.61) and a sensitivity of $35.2 \%$ (95\% CI 26.944.1 ), a specificity of $98.6 \%$ (95\% CI 98.5-98.7), a DOR of 14.7 (95\% CI 4.1-52.5), an LR+ of 9.6 (2.7-33.5), and an LR- of 0.7 (95\% CI 0.6-0.9) (Fig. 3). Table 3 shows the figures for diagnostic accuracy in low- and high-risk populations. Detection of TR at the 11-14 weeks' scan showed an LR+ of 7.2 (95\% CI 5.3-9.8) in detecting CHD when applied to a population at risk for CHD such as fetuses with an increased NT. Diagnostic performance was poor in a low-risk population (Table 3 ).
$4 \quad$ Fetal Diagn Ther 2017;42:1-8
Scala et al. 
Table 3. Summary estimates of sensitivity, specificity, positive and negative likelihood ratios (LR+ and LR-), and diagnostic odds ratio (DOR) of tricuspid valve regurgitation in the first trimester for the detection of congenital heart disease

\begin{tabular}{|c|c|c|c|c|c|c|c|}
\hline & $\begin{array}{l}\text { Studies, } \\
n\end{array}$ & $\begin{array}{l}\text { Total } \\
\text { sample, } n\end{array}$ & $\begin{array}{l}\text { Sensitivity, \% } \\
(95 \% \text { CI) }\end{array}$ & $\begin{array}{l}\text { Specificity, \% } \\
(95 \% \text { CI })\end{array}$ & $\begin{array}{l}\text { DOR } \\
(95 \% \text { CI })\end{array}$ & $\begin{array}{l}\mathrm{LR}+ \\
(95 \% \mathrm{CI})\end{array}$ & $\begin{array}{l}\text { LR- } \\
(95 \% \mathrm{CI})\end{array}$ \\
\hline Overall & 3 & 42,451 & $35.2(26.9-44.1)$ & $98.6(98.5-98.7)$ & $14.7(4.1-52.5)$ & $9.6(2.7-33.5)$ & $0.7(0.6-0.9)$ \\
\hline High-risk population & 2 & 1,154 & $53.4(39.9-66.7)$ & $92.2(90.4-93.7)$ & $15.2(8.5-27.2)$ & $7.2(5.3-9.8)$ & $0.5(0.4-0.7)$ \\
\hline Low-risk population & 2 & 41,993 & $31.3(22.2-41.5)$ & $98.6(98.5-98.7)$ & $12.5(0.9-174.1)$ & $9.3(0.8-111.8)$ & $0.75(0.6-0.96)$ \\
\hline
\end{tabular}

Computations were based upon DerSimonian-Laird random effect.

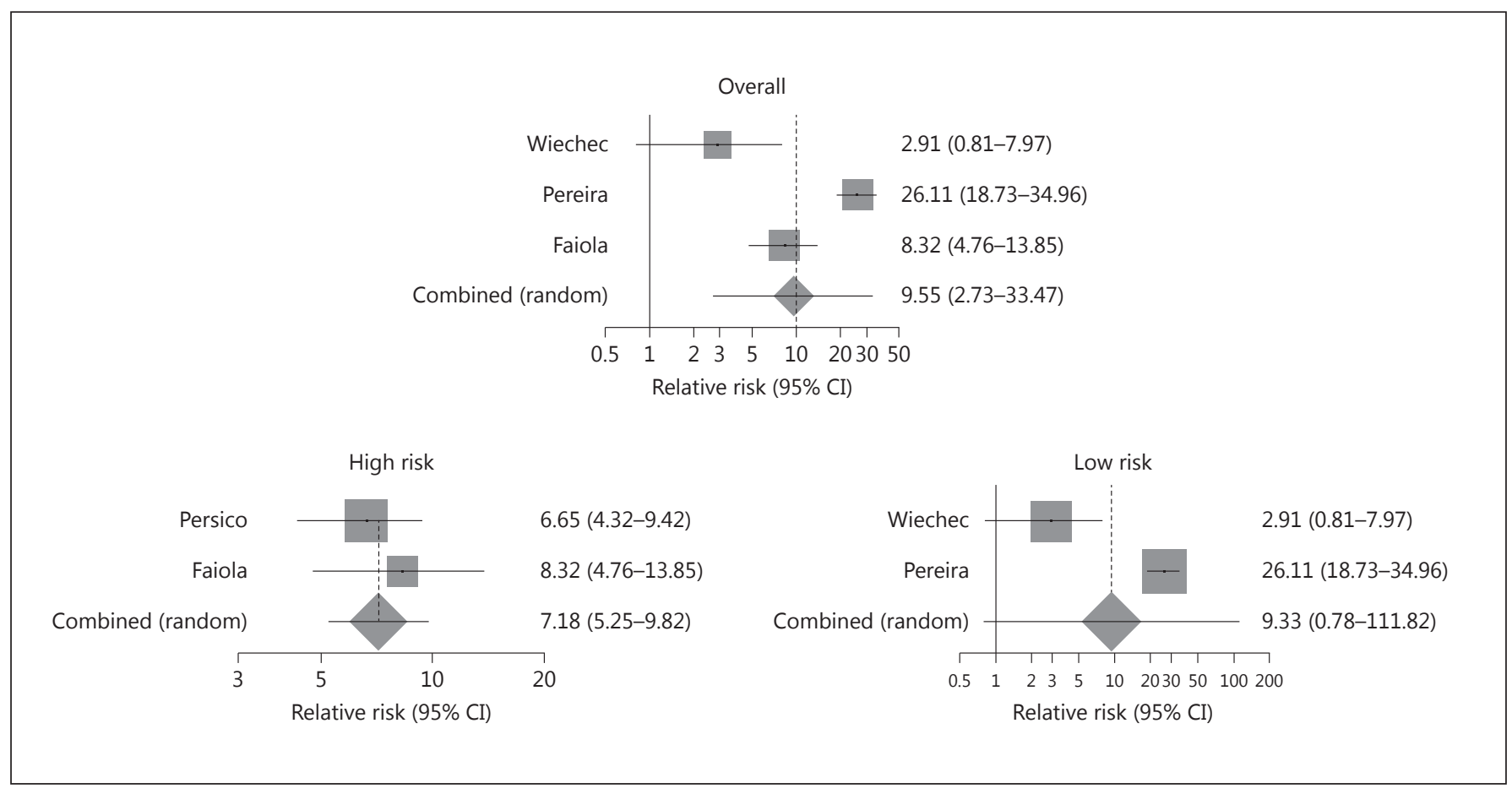

Fig. 2. Forest plot showing the relative risk of congenital heart disease in euploid fetuses with tricuspid regurgitation detected in the first trimester of pregnancy.

\section{Discussion}

\section{Main Findings}

The findings from this systematic review showed that TR detected during the 11-14 weeks' scan increased the risk of CHD. However, this association is higher for cases considered to be at high risk for cardiac defects, such as those with increased NT, while there is no association between TR and CHD in a low-risk population. Diagnostic accuracy of TR in detecting CHD was poor, although TR performed better when applied to fetuses at higher risk for CHD such as those with increased NT.

Fetal TR in the First Trimester as a

Screening Marker for CHD

\section{Strength and Limitations}

This is the first systematic review investigating the predictive accuracy of TR in the first trimester as an isolated marker for CHD. This systematic review has a comprehensive search strategy and provides quantitative pooling of the data using meta-analysis of proportion and HSROC. The method used to assess TR was the same in all of the included studies and was performed by sonographers or medical doctors who had received the appropriate Fetal Medicine Certificate of competence. Heterogeneity in the populations analyzed in each study represents the major limitations of this systematic review. The published prev-

Fetal Diagn Ther 2017;42:1-8 DOI: $10.1159 / 000455947$ 


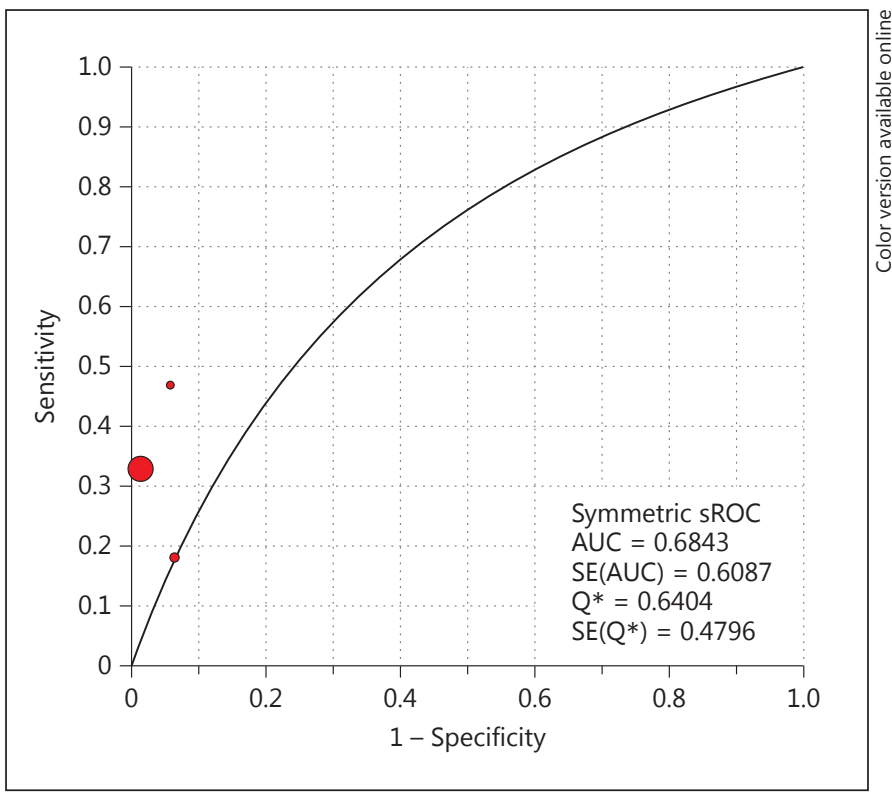

Fig. 3. Summary receiver-operating characteristics curves (sROC) showing the diagnostic performance of tricuspid regurgitation in the first trimester for the detection of congenital heart disease. Computations were based upon DerSimonian-Laird random effect.

alence of TR in fetuses with CHD varies widely, ranging from 18 to $62 \%$ [20-23]. One of the main factors influencing this variability is the nature of the pregnancies screened, whether low- or high-risk. However, our metaanalysis showed that the LR+ of CHD in the presence of TR was approximately the same in the two subpopulations (9 and 10 for the low- and high-risk populations, respectively). In fact, even though the pooled RR in the low-risk population was apparently higher than in the high-risk population, this result was not significantly different ( $p=0.057)$. This might be due to the small number of studies included in the analysis and the high level of heterogeneity.

\section{Interpretation of the Findings}

This meta-analysis showed that TR, when detected in the first trimester, is a significant risk factor for CHD in euploid fetuses (LR+ $=13$ ). Furthermore, normal tricuspid blood flow is a significant protective marker $(\mathrm{LH}-=$ $0.4)$. It has been widely demonstrated that first-trimester indirect signs, including NT, TR, ductus venosus (DV), and abnormal cardiac axis, are significantly associated with the risk of CHD $[24,25]$. The underlying mechanism of this association is uncertain. However, these markers could be a consequence of cardiac impairment, which is manifest only during the first trimester of pregnancy when the compliance of the fetal heart is low and the placental resistance is high [22].

\section{Clinical and Research Implications}

Although the assessment of TR in the first trimester is achievable in approximately $98 \%$ of cases [26], it is unlikely that it will be routinely used to screen for CHD in a first-level low-risk screening setting. It is more likely to be used in tertiary fetal medicine units. According to the Fetal Medicine Foundation, the assessment of tricuspid flow need not be carried out in all pregnancies undergoing routine first-trimester combined screening. Such examination could be reserved for the $15 \%$ of the total population with an intermediate risk (between 1 in 51 and 1 in $1,000)$ after combined testing.

Our meta-analysis shows that TR alone is not a strong predictor of CHD. However, it could represent an indirect way of fetal heart assessment for identifying those fetuses at an increased risk of CHD. The decision to perform further cardiac evaluation in cases with normal karyotype and TR should be prompted by an individualized risk assessment incorporating all available risk factors.

Two approaches have been proposed for the use of the algorithm combining NT, DV Doppler, and TR to estimate the patient-specific risk of major CHD. The first is to define the risk cut-off that selects patients requiring referral for specialist fetal echocardiography. The risk increases exponentially with NT thickness from 1 per 1,000 in those with NT at or below the 95th centile to 7 per 1,000 for NT between the 95th and 99th centile, and 58 per 1,000 for NT above the 99th centile. The risk is further increased if there is DV reversed a-wave, TR, or both, and is decreased if flow in the DV and across the tricuspid valve is normal. The second approach is to define as highrisk all cases with TR, DV reversed a-wave, or both, which constitute approximately $3 \%$ of the population and contain $48 \%$ of those with major cardiac defects. If cases with NT above the 99th centile are also included, the screenpositive rate would increase to approximately $4 \%$ and the estimated detection rate to $52 \%$. If there are sufficient resources to perform fetal echocardiography in $8 \%$ of the population, then the NT cut-off for defining the high-risk group could be reduced to the 95th percentile with an increase in the estimated detection rate to 58\% [20].

Another approach is the direct evaluation of the fetal heart; several studies have reported that a major CHD can be detected during the first trimester of pregnancy by experienced operators in both high-risk and unselected 
populations [20-27]. The early detection rate for the most common cardiac abnormalities varied from around 51\% for hypoplastic left heart to $16 \%$ for coarctation of the aorta and 18\% for tetralogy of Fallot and transposition of the great arteries [28]. However, in accordance with the International Society of Ultrasound in Obstetrics and Gynecology safety statement, Doppler examination of fetal vessels in early pregnancy should not be performed without a clinical indication. Teaching and training Doppler sessions on first-trimester fetuses should be kept short and should only be performed in fetuses in whom Doppler examination is indicated anyway and not in the general population $[29,30]$.

The largest study, involving 44,859 singleton pregnancies undergoing first-trimester ultrasound scan as part of routine screening for aneuploidies, reported that the detection rate of major CHD was 34\% [7]. The study reported that this scan led to the diagnosis of around half of cases of double outlet right ventricle, hypoplastic left heart, and transposition of the great arteries, around onethird of cases of atrioventricular septal defect, coarctation of the aorta, tetralogy of Fallot, and pulmonary atresia, but none of the cases of ventricular septal defect, Ebstein anomaly, aortic or pulmonary stenosis, tricuspid atresia, or cardiac tumors. A recent review of the published series with more than 1,000 cases from 1993 to 2008, which included data from 36,237 pregnancies generated by eight centers, suggests that the overall detection rate of major $\mathrm{CHD}$ at $11-13$ weeks is $29 \%$ (95\% CI $25-33)$ [6]. The pooled detection rate of cardiac defects was 17\% (10-25). The authors suggested that the detection rate could be improved if the first-trimester ultrasound assessment followed very well-delineated protocols [31].

Currently, there are no recommendations to perform routine detailed fetal cardiac evaluation when TR is detected in the first trimester. However, in such cases, fetal medicine specialists should consider performing a detailed ultrasound evaluation of other markers of $\mathrm{CHD}$ and direct evaluation of the fetal heart. If additional markers, such as reversed a-wave in the DV or increased NT, are present or there is suspicion of major CHD, early fetal echocardiography should be offered.

\section{Conclusion}

In conclusion, detection of TR in the first trimester increases the risk of CHD. However, isolated TR in the first trimester does not seem to be a strong predictor of CHD. Prospective large screening studies are necessary to create an individualized adjusted risk model taking into account known first-trimester risk factors, in order to improve the antenatal detection of CHD.

\section{Disclosure Statement}

The authors report no conflict of interest. No specific funding was obtained for this study.

\section{References}

1 Mensah GA, Brown DW: An overview of cardiovascular disease burden in the United States. Health Affairs 2007;26:38-48.

2 Li Y, Hua Y, Fang J, Wang C, Qiao L, Wan C, Mu D, Zhou K: Performance of different scan protocols of fetal echocardiography in the diagnosis of fetal congenital heart disease: a systematic review and meta-analysis. PLoS One 2013;8:e65484

3 MacColl CE, Manlhiot C, Page C, McCrindle BW, Miner SE, Jaeggi ET, Nield LE: Factors associated with in utero demise of fetuses that have underlying cardiac pathologies. Pediatr Cardiol 2014:35:1403-1414.

4 Fesslova' V, Brankovic J, Boschetto C, Masini A, Prandstraller D, Perolo A, Ventriglia F, Macerola S, Crepaz R, Romeo C, De Luca F, Previtera A, Errico G; Fetal Study Group of Italian Society of Pediatric Cardiology: Changed outcomes of fetuses with congenital heart disease: new Italian multicentre study. J Cardiovasc Med (Hagerstown) 2015;16:568575 .

Fetal TR in the First Trimester as a

Screening Marker for CHD
5 van Velzen CL, Clur SA, Rijlaarsdam M, Bax CJ, Pajkrt E, Heymans MW, Bekker MN, Hruda J, de Groot C, Blom NA, Haak MC: Prenatal detection of congenital heart disease-results of a national screening programme. BJOG 2016;123:400-407.

6 Hildebrand E, Selbing A, Blomberg M: Comparison of first and second trimester ultrasound screening for fetal anomalies in the southeast region of Sweden. Acta Obstet Gynecol Scand 2010;89:1412-1419.

7 Syngelaki A, Chelemen T, Dagklis T, Allan L, Nicolaides KH: Challenges in the diagnosis of fetal non-chromosomal abnormalities at 1113 weeks. Prenat Diagn 2011;31:90e102.

8 Huggon IC, DeFigueiredo DB, Allan LD: Tricuspid regurgitation in the diagnosis of chromosomal anomalies in the fetus at 11-14 weeks of gestation. Heart 2003;89:10711073.

9 Ghaffari SR, Tahmasebpour AR, Jamal A, Hantoushzadeh S, Eslamian L, Marsoosi V, Fattahi F, Rajaei M, Niroomanesh S, Borna S,
Beigi A, Khazardoost S, Saleh-Gargari S, Rahimi-Sharbaf F, Farrokhi B, Bayani N, Tehrani SE, Shahsavan K, Farzan S, Moossavi S, Ramezanzadeh F, Dastan J, Rafati M: Firsttrimester screening for chromosomal abnormalities by integrated application of nuchal translucency, nasal bone, tricuspid regurgitation and ductus venosus flow combined with maternal serum free $\beta$-hCG and PAPP-A: a 5 -year prospective study. Ultrasound Obstet Gynecol 2012;39:528-534.

10 Falcon O, Faiola S, Huggon I, Allan L, Nicolaides $\mathrm{KH}$ : Fetal tricuspid regurgitation at the $11+0$ to $13+6$-week scan: association with chromosomal defects and reproducibility of the method. Ultrasound Obstet Gynecol 2006;27:609-612.

11 Stroup DF, Berlin JA, Morton SC, et al: Metaanalysis of observational studies in epidemiology: a proposal for reporting. Meta-analysis Of Observational Studies in Epidemiology (MOOSE) group. JAMA 2000;283:20082012.
Fetal Diagn Ther 2017;42:1-8 DOI: $10.1159 / 000455947$ 
12 von Elm E, Altman DG, Egger $M$, et al; STROBE Initiative: The Strengthening the Reporting of Observational Studies in Epidemiology (STROBE) statement: guidelines for reporting observational studies. Lancet 2007; 370:1453-1457.

13 Leeflang MM, Deeks JJ, Gatsonis C, Bossuyt PM; Cochrane Diagnostic Test Accuracy Working Group: Systematic reviews of diagnostic test accuracy. Ann Intern Med 2008; 149:889-897.

14 Messing B, Porat S, Imbar T, Valsky DV, Anteby EY, Yagel S. Mild tricuspid regurgitation: a benign fetal finding at various stages of pregnancy. Ultrasound Obstet Gynecol 2005;26: 606-609.

15 Rutter CM, Gatsonis CA: A hierarchical regression approach to metaanalysis of diagnostic test accuracy evaluations. Stat Med 2001;20:2865-2884.

16 Cochrane Handbook for Systematic Reviews of Diagnostic Test Accuracy, Chapter 10; http://srdta.cochrane.org/handbook-dta-reviews.

17 Glas AS, Lijmer JG, Prins MH, et al: The diagnostic odds ratio: a single indicator of test performance. J Clin Epidemiol 2003;56:11291135.

18 Higgins JP, Thompson SG: Quantifying heterogeneity in a meta-analysis. Stat Med 2002; 21:1539-1558.

19 Song F, Khan KS, Dinnes J, Sutton AJ: Asymmetric funnel plots and publication bias in meta-analyses of diagnostic accuracy. Int J Epidemiol 2002;31:88-95.
20 Persico N, Moratalla J, Lombardi CM, Zidere V, Allan L, Nicolaides KH: Fetal echocardiography at 11-13 weeks by transabdominal high-frequency ultrasound. Ultrasound $\mathrm{Ob}-$ stet Gynecol 2011;37:296-301.

21 Faiola S, Tsoi E, Huggon IC, Allan L, Nicolaides KH: Likelihood ratio for trisomy 21 in fetuses with tricuspid regurgitation at the 11 to 13+6-week scan. Ultrasound Obstet Gynecol 2005;26:22-27.

22 Wiechec M, Nocun A, Wiercinska E, Beithon J, Knafel A: First trimester tricuspid regurgitation and fetal abnormalities. J Perinat Med 2015;43:597-603.

23 Pereira S, Ganapathy R, Syngelaki A, Maiz N, Nicolaides KH: Contribution of fetal tricuspid regurgitation in first-trimester screening for major cardiac defects. Obstet Gynecol 2011;117:1384-1391.

24 Volpe P, Ubaldo P, Volpe N, Campobasso G, De Robertis V, Tempesta A, Volpe G, Rembouskos G: Fetal cardiac evaluation at 11-14 weeks by experienced obstetricians in a lowrisk population. Prenat Diagn 2011;31:10541061.

25 Sinkovskaya ES, Chaoui R, Karl K, Andreeva E, Zhuchenko L, Abuhamad AZ: Fetal cardiac axis and congenital heart defects in early gestation. Obstet Gynecol 2015;125:453-460.

26 Karadzov-Orlic N, Egic A, Milovanovic Z, Marinkovic M, Damnjanovic-Pazin B, Lukic R, Joksic I, Curkovic A, Mikovic Z: Improved diagnostic accuracy by using secondary ultrasound markers in the first-trimester screening for trisomies 21, 18 and 13 and Turner syndrome. Prenatal Diagnosis 2012;32:638643.
27 Bellotti M, Fesslova V, De Gasperi C, Rognoni G, Bee V, Zucca I, Cappellini A, Bulfamante G, Lombardi CM: Reliability of the firsttrimester cardiac scan by ultrasound-trained obstetricians with high-frequency transabdominal probes in fetuses with increased nuchal translucency. Ultrasound Obstet Gynecol 2010;36:272-278.

28 Khalil A, Nicolaides KH: Fetal heart defects: potential and pitfalls of first-trimester detection. Semin Fetal Neonatal Med $2013 ; 18$ : 251-260.

29 Salvesen K, Lees C, Abramowicz J, Brezinka C, Ter Haar G, Maršál K; Board of International Society of Ultrasound in Obstetrics and Gynecology (ISUOG): ISUOG statement on the safe use of Doppler in the 11 to 13+6-week fetal ultrasound examination. Ultrasound Obstet Gynecol 2011;37:628.

30 Salomon LJ, Alfirevic Z, Bilardo CM, Chalouhi GE, Ghi T, Kagan KO, Lau TK, Papageorghiou AT, Raine-Fenning NJ, Stirnemann J, Suresh S, Tabor A, Timor-Tritsch IE, Toi A, Yeo G: ISUOG practice guidelines: performance of first-trimester fetal ultrasound scan. Ultrasound Obstet Gynecol 2013;41: 102-113.

31 Borrell A, Robinson JN, Santolaya-Forgas J: Clinical value of the 11- to 13+6-week sonogram for detection of congenital malformations: a review. Am J Perinatol 2011;28:117124. 